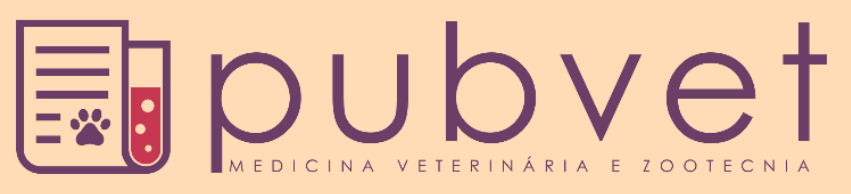

https://doi.org/10.31533/pubvet.v14n9a658.1-7

\title{
Citronela, Neen e Noni no controle de Riphicephalus (Boophilus) microplus in vitro
}

\author{
${ }^{I}$ Discente do Curso de Zootecnia, Faculdades Associadas de Uberaba, Uberaba-MG Brasil. \\ ${ }^{2}$ Professor do Curso de Medicina Veterinária, Universidade de Uberaba, Uberaba - MG Brasil. \\ ${ }^{3}$ Professora do Curso de Farmácia, Universidade de Uberaba, Uberaba-MG Brasil. \\ ${ }^{4}$ Professora do Curso de Zootecnia, Faculdades Associadas de Uberaba, Uberaba-MG Brasil. \\ *Autor para correspondência, E-mail: apnquintal@yahoo.com.br
}

Alan Brudniewski ${ }^{19}$, André Belico de Vasconcelos ${ }^{2} \oplus \odot$, Elisabeth Bucek ${ }^{3}$, Amanda Pifano Neto Quintal ${ }^{4}$

Resumo. O tratamento químico do carrapato vem sendo amplamente utilizado, porém de forma indiscriminada, levando ao aumento da ocorrência de resistência aos fármacos sintéticos por parte desse parasita. Na tentativa de controle do Ripicephalus (Boophilus) microplus, o presente trabalho objetivou avaliar a eficácia de tratamentos fitoterápicos como alternativas medicamentosas empregadas em seu controle. Para a realização do experimento, foram coletadas 500 teleógenas de uma fazenda sem tratamento farmacológico durante 60 dias e posteriormente foram submetidas ao teste de biocarrapaticidograma a fim de se avaliar a eficiência reprodutiva e eficácia medicamentosa de cada tratamento. Posteriormente, foi realizado o teste de imersão de larvas, avaliando-se o efeito larvicida dos tratamentos. Para os tratamentos foram usados (1) extrato hidroalcoólico de citronela, (2) óleo emulsionável comercial de neen, (3) extrato hidroalcoólico de noni, (4) produto comercial cipermetrina e (5) água. O extrato hidroalcoólico de citronela destacou-se frente aos demais tratamentos fitoterápicos com $50 \%$ de eficiência e a cipermetrina foi $100 \%$ eficaz no controle do $R$. (B.) microplus in vitro (Anova $\mathrm{P}>0,05$ ). Este cenário foi semelhante ao protocolo larval (Anova $\mathrm{P}>0,05$ ). Destaca-se a importância de alternância de princípios ativos, o que se pode incluir o uso de fitoterápicos, como o extrato hidroalcoólico de citronela, de forma preventiva; somados a aplicação da cipermetrina em pontos estratégicos em animais altamente infestados. Este manejo pode ser útil no controle do carrapato bovino, destacando ainda a importância de uso de extrato de citronela em criações orgânicas.

Palavras chave: Ectoparasita, fitoterápicos, biocarrapaticidograma. teste de imersão de larvas

\section{Citronella, Neen and Noni for Ripicephalus (Boophilus) microplus control in vitro}

Abstract. The chemical tick treatment has been widely used, however in an indiscriminate way, leading to resistance increase to synthetic drugs by this parasite. In an attempt to control Ripicephalus (Boophilus) microplus, the present study aimed to evaluate the effectiveness of herbal treatments as alternative in their control. For the experiment, 500 teleogenes were collected from a farm without pharmacological treatment during 60 days and were subsequently submitted to biocarrapaticidogram test to evaluate the reproductive efficiency and drug efficacy of the treatments. Subsequently, the larvae immersion test was performed, evaluating the treatments larvicidal effect. For the treatments were used: (1) citronella hydroalcoholic extract, (2) neen commercial emulsifiable oil, (3) noni hydroalcoholic extract, (4) commercial cypermethrin product and (5) water. The hydroalcoholic citronella extract stood out compared to other herbal treatments with 50\% efficiency and cypermethrin was $100 \%$ effective in controlling $R$. (B.) microplus in vitro 
(Anova $\mathrm{P}>0.05$ ). This scenario was similar to larval protocol (Anova $\mathrm{P}>0.05$ ). It highlight the alternating active medicaments importance, which may include herbal medicines use, such as hydroalcoholic citronella extract, in a preventive way; plus the cypermethrin application at strategic points in infested animals. This management can be useful in bovine tick control, also highlighting the using citronella extract importance in organic farms.

Keywords: Ectoparasite, phytotherapy, Biocarrapaticidogram. Immersion larvae test

\section{Citronela, Neen y Noni en el control de Ripicephalus (Boophilus) microplus in vitro}

Resumen. El tratamiento químico de la garrapata se ha utilizado ampliamente, sin embargo, de manera indiscriminada, lo que lleva a un aumento de la resistencia a los fármacos sintéticos por este parásito. En un intento por controlar Ripicephalus (Boophilus) microplus, el presente estudio tuvo como objetivo evaluar la efectividad de los tratamientos a fitoterapéuticos como medicamentos alternativos utilizados en su control. Para llevar a cabo el experimento, se recogieron 500 garrapatas hembra de una granja sin tratamiento farmacológico durante 60 días y posteriormente se sometieron a biocarrapaticidograma para evaluar la eficiencia reproductiva y la eficacia del fármaco de cada tratamiento. Posteriormente, se realizó la prueba de inmersión en larvas, evaluando el efecto larvicida de los tratamientos. Para los tratamientos (1) extracto hidroalcohólico de citronela, (2) aceite emulsionable comercial neen, (3) extracto hidroalcohólico noni, (4) producto de cipermetrina comercial y (5) agua. El extracto de citronela hidroalcohólico se destacó en comparación con otros tratamientos herbales con un $50 \%$ de eficiencia y la cipermetrina fue $100 \%$ efectiva en el control de $R$. (B.) microplus in vitro (Anova $\mathrm{P}>0,05$ ). Este escenario fue similar al protocolo larval (Anova $\mathrm{P}>0,05$ ). Se destaca la importancia de alternar ingredientes activos, que pueden incluir el uso de medicamentos a base de hierbas, como el extracto de citronela hidroalcohólico, de forma preventiva; más la aplicación de cipermetrina en puntos estratégicos en animales altamente infestados. Este manejo puede ser útil en el control de la garrapata bovina, destacando también la importancia del uso de extracto de citronela en creaciones orgánicas.

Palabras clave: Ectoparásito, fitoterápicos, biocarrapaticidograma, prueba de inmersión en larvas

\section{Introdução}

O carrapato que acomete bovinos, Ripicephalus (Boophilus) microplus, é um dos principais ectoparasitas presentes no Brasil cuja infestação pelo carrapato provoca prejuízos significativos à bovinocultura mundial, causando grande impacto econômico com perda de dezenas de bilhões de dólares ao ano em todo mundo, provocando danos diretos e indiretos à atividade (Andreotti et al., 2015). Perdas no Brasil podem chegar a mais de 3,24 bilhões de dólares anuais (Oliveira \& Pedrassani, 2017).

O carrapato bovino atua como vetor de patógenos causadores por doenças como Babesiose e Anaplasmose (Ríos-Tobón et al., 2014), onde os hemoprotozoários Anaplasma marginale, Babesia bovis e Babesia bigemina utilizam-se do carrapato para disseminarem suas respectivas enfermidades.

$\mathrm{Na}$ tentativa de evitar tais prejuízos, grande parte dos produtores brasileiros têm utilizado carrapaticidas como método de controle desse parasita, porém como ainda não existe qualquer política oficial de controle ao carrapato bovino a saída encontrada por muitos tem sido adotar práticas de controle individuais (Gomes et al., 2011; Oliveira \& Pedrassani, 2017).

Tanto o controle corretivo quanto preventivo, os carrapaticidas são amplamente utilizados no controle desse ectoparasita, porém são cada vez menos eficientes devido a forma incorreta e indiscriminada de uso, o que pode acelerar resistência de compostos químicos utilizados ( $\underline{\text { Santos et al., 2006). }}$.

Assim, constatou-se a necessidade de novas alternativas de controle com as quais o carrapato não apresente mecanismos de resistência, gerando menores ricos à saúde humana e redução do impacto ambiental, minimizando as perdas econômicas por parte dos produtores (Oliveira \& Pedrassani, 2017). 
A utilização de fitoterápicos tem sido considerada (Borges et al., 2011), portanto, este trabalho objetivou gerar alternativas fitoterápicas Neen, Noni e Citronela no controle do carrapato bovino Rhipicephalus (Boophilus) microplus in vitro.

\section{Material e métodos}

Foram coletadas 500 teleógenas, cujos animais estavam sem tratamento químico por mais de 60 dias em todas as ocasiões de coleta, em uma fazenda na Região de Uberaba-MG. Os carrapatos foram alocados em caixa de isopor e transportados ao laboratório de microbiologia da FAZU.

No laboratório, as teleógenas foram pesadas em balança de precisão e separadas por homogeneidade de peso, alojando cinco carrapatos por placa de Petri, totalizando 20 placas por grupo de tratamento.

Para o tratamento, os carrapatos foram submetidos aos banhos de água (controle) e os medicamentos separados para avaliação foram a cipermetrina, extrato hidroalcoólico de citronela, extrato de neen e extrato hidroalcoólico de noni.

A base química de Cipermetrina (CIPERMETRINA 250CE Colosso ${ }^{\circledR}$, Ouro Fino), utilizada na Fazenda Escola da FAZU na forma de pour on, contendo para cada $100 \mathrm{~mL} 15 \mathrm{~g}$ de cipermetrina, $25 \mathrm{~g}$ de clorpirifos e $1 \mathrm{~g}$ de citronela, diluídos 1 litro de colosso para 500 litros de água, conforme recomendações do fabricante.

O extrato comercial de óleo de neen $\left(\right.$ Vitaplan $^{\circledR}$ ), foi diluído $5 \mathrm{~mL}$ para cada $500 \mathrm{~mL}$ de água, cujas concentrações de acordo com a recomendação do fabricante.

O extrato hidroalcoólico de citronela (Cymbopogon citratus), apresentando o citronelal, citronelol e geranial como seus principais princípios ativos (Agnolin et al., 2014) produzido no setor de produção de leite orgânico da Fazenda Escola da FAZU, pela solubilização das folhas de citronela em etanol durante quinze dias, sendo adicionados dois litros de solução alcoólica em cinco litros de água, tornando o extrato hidroalcoólico de citronela pronto para o uso.

O extrato hidroalcoólico de noni (Morinda citrifolia) foi obtido a partir da coleta aleatória das plantas de noni cujos derivados vegetais (sumo, sucos e extratos) foram preparados a partir dos órgãos frescos (sumo: por expressão, e armazenamento a $-20^{\circ} \mathrm{C}$ ) e/ou secos e a partir de drogas vegetais de fruto e folha, cujos extratos foram obtidos com solventes, etanol, água e solução hidro alcoólica de acordo com Correia et al. (2011), feito no laboratório de Fitopatologia da Universidade de Uberaba.

Após estabelecidos os tratamentos e preparadas as soluções, foi realizado o banho de imersão das fêmeas em solução acaricida por cinco minutos, seguida por secagem dos carrapatos em papel toalha e realocadas em placas de Petri, colocadas em estufa a $37^{\circ} \mathrm{C}$ e monitoradas diariamente, por 15 dias para verificação do índice de eclosão através da pesagem dos ovos eclodidos por cada fêmea.

Os ovos pesados foram pesados, separados e colocados em tubos de polipropileno com rosca, em estufa a $37^{\circ} \mathrm{C}$. Após 15 dias foi estimado o percentual de eclosão dos ovos com a presença de larvas nos tubos, bem como os cálculos de eficiência reprodutiva e eficiência medicamentosa dos tratamentos (Drummond et al., 1973) , mensurados pelas seguintes equações: ER $=($ Peso dos ovos $) /($ Peso das teleógenas) $\mathrm{x} \%$ Eclosão $\times 20.000$ e ET = (ER do grupo controle - ER do grupo tratado)/ (ER do grupo controle) x 100.

Após a eclosão das larvas estas foram colocadas em tubo de prolipropileno com tampa rosca e imersas em solução carrapaticida, cada uma em seu respectivo princípio ativo, durante 5 minutos. Posteriormente, este conteúdo foi filtrado em papel filtro, possibilitando a contagem de larvas vivas e mortas pelo movimento das mesmas (Sousa et al., 2008). Assim, pôde ser verificada a taxa de mortalidade larval, observando-se o efeito do medicamento nesta fase do ciclo biológico do parasita.

Para análise estatística, os dados foram expressos como média \pm desvio padrão (DP) dos tratamentos diagnósticos, utilizando-se o programa Graph Pad Prism 6.0 (GraphPad Software Inc). O delineamento utilizado foi inteiramente casualizado, tendo medicamentos como tratamentos e única causa de variação. Eficiência reprodutiva, eficiência do produto e mortalidade de larvas foram expressas em porcentagem e avaliados por análise de variância (ANOVA) seguida pelo teste de Tukey com significância de 5\%. 


\section{Resultados}

Os resultados coletados durante a realização do biocarrapaticidograma estão dispostos na Tabela 1. Importante destacar que o peso das teleógenas foi padronizado entre os tratamentos, não sendo observado a diferença no peso das teleógenas (Anova P > 0,05).

Tabela 1. Peso das teleógenas, peso dos ovos eclodidos e taxa de eclosão de ovos coletados durante o biocarrapaticidograma.

\begin{tabular}{lccc}
\hline \multirow{2}{*}{ Tratamentos } & \multicolumn{3}{c}{ Parâmetros do biocarrapaticidograma } \\
\cline { 2 - 4 } & $\begin{array}{c}\text { Peso das Teleógenas, em } \\
\text { gramas }^{1}\end{array}$ & $\begin{array}{c}\text { Peso dos ovos eclodidos, em } \\
\text { gramas }^{2}\end{array}$ & $\begin{array}{c}\text { Taxa de eclosão dos ovos, em } \\
\text { percentual }^{3}\end{array}$ \\
\hline Água & $0,58 \pm 0,27^{\mathrm{a}}$ & $0,20 \pm 0,14^{\mathrm{a}}$ & $94,75 \% \pm 3,8^{\mathrm{a}}$ \\
Cipermetrina & $0,55 \pm 0,29^{\mathrm{a}}$ & $0 \pm 0^{\mathrm{b}}$ & $0 \% \pm 0^{\mathrm{b}}$ \\
Citronela & $0,56 \pm 0,30^{\mathrm{a}}$ & $0,19 \pm 0,20^{\mathrm{a}}$ & $84,42 \% \pm 11,1^{\mathrm{a}}$ \\
Neen & $0,55 \pm 0,30^{\mathrm{a}}$ & $0,21 \pm 0,17^{\mathrm{a}}$ & $90,1 \% \pm 10,7^{\mathrm{a}}$ \\
Noni & $0,58 \pm 0,29^{\mathrm{a}}$ & $0,18 \pm 0,18^{\mathrm{a}}$ & $94,1 \% \pm 5,3^{\mathrm{a}}$ \\
\hline
\end{tabular}

${ }^{1}$ Peso das teleógenas (Anova/Tukey P $\left.>0,05\right) .{ }^{2}$ Peso dos ovos eclodidos (Anova/Tukey P $\left.<0,05\right) .{ }^{3}$ Taxa de eclosão de ovos (Anova/Tukey $\mathrm{P}<0,05)$.

Para a análise do peso dos ovos eclodidos, houve diferença entre os pesos dos grupos imersos em cipermetrina comparados aos demais tratamentos (Anova $\mathrm{P}>0,05$ ), evidenciando a eficácia do tratamento químico, atuando diretamente no controle das teleógenas adultas e, consequentemente, impedindo a oviposição. Somados, observou-se a ausência de resistência a cipermetrina nas propriedades da região. Não houve diferença quanto ao peso dos ovos comparando o tratamento controle com os tratamentos fitoterápicos (Anova $\mathrm{P}>0,05$ ).

Para avaliar a taxa de eclosão das larvas, o extrato hidroalcoólico de citronela foi o tratamento fitoterápico que mais reduziu a eclosão dos ovos em larvas, seguido pelo extrato de noni, extrato de neen. Como não houve postura de ovos em teleógenas com cipermetrina, consequentemente não houve a pesagem e nem eclosão de seus ovos, sendo valores zero.

Vale destacar que, das 100 teleógenas avaliadas por tratamento, eclodiram 99\% (99/100) para água, $79 \%$ (79/100) para citronela; 85\% (85/100) para neen, 89\% (89/100) para noni e 0\% (0/100) para cipermetrina.

A eficiência reprodutiva (Figura 1A) diferiu entre os tratamentos $(\mathrm{P}<0,05)$, tendo o tratamento químico causado a morte de todas as teleógenas. Entre os tratamentos fitoterápicos, o extrato de citronela se destacou frente aos demais, apesar dos grupos apresentarem um desvio considerável entre as placas.

A eficiência total medicamentosa (Figura 1B) foi calculada de acordo com os valores de eficiência reprodutiva encontrados no grupo controle e o tratamento avaliado. Pode-se observar uma baixa eficiência dos extratos neen e noni; uma eficiência intermediária do extrato de citronela e o controle químico a base de cipermetrina apresentou $100 \%$ de eficiência, uma vez que controlou a população testada antes que estas realizassem a postura dos ovos $(\mathrm{P}<0,05)$.
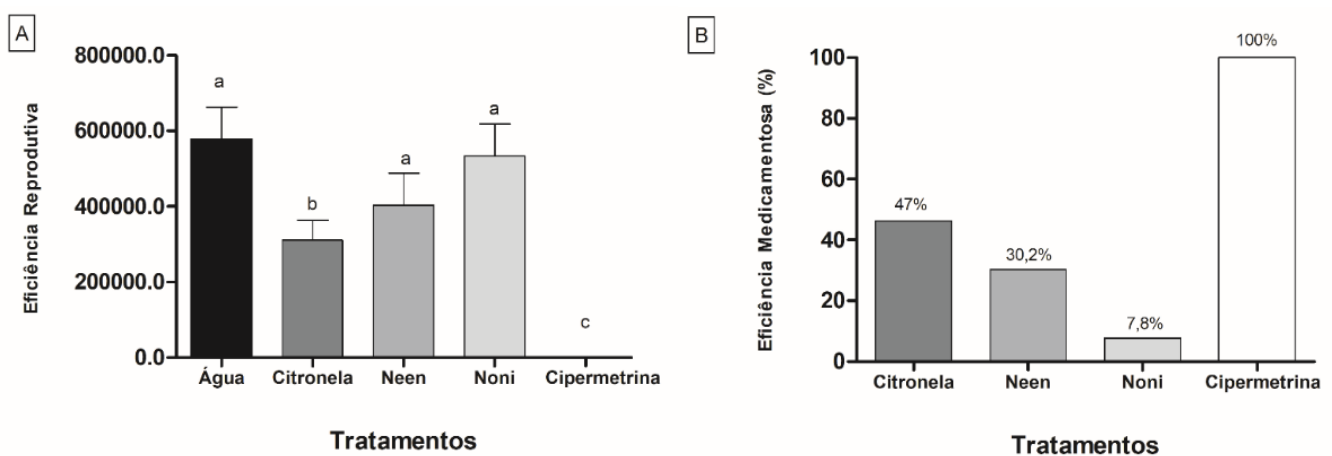

Figura 1. Eficiência reprodutiva (A) e eficiência medicamentosa (B) dos tratamentos fitoterápicos e químico para o controle de carrapatos bovinos pelo teste de biocarrapaticidograma in vitro. 
Durante o teste de imersão das larvas (Figura 2), a cipermetrina demonstrou eficácia e o extrato hidroalcóolico de citronela demonstrou maior eficiência larvicida frente aos demais tratamentos fitoterápicos $(\mathrm{P}>0,05)$.

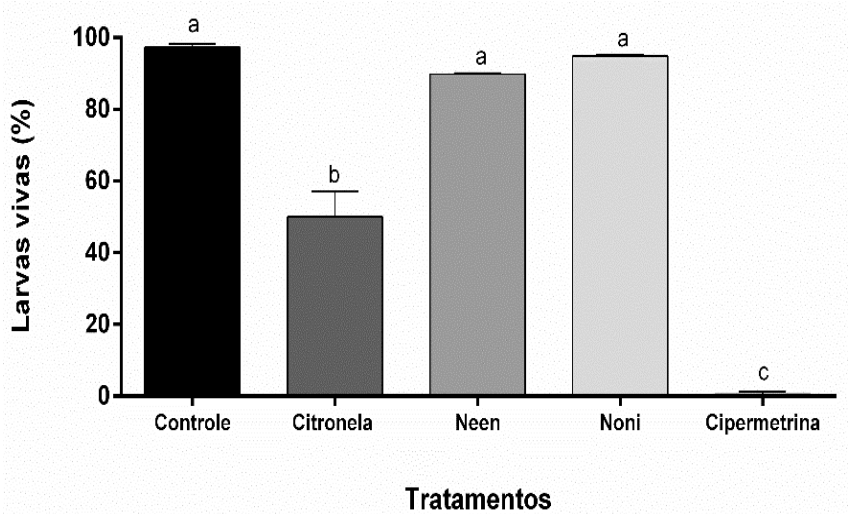

Figura 2. Teste de imersão de larvas dos tratamentos fitoterápicos e químico para o controle de carrapatos bovinos.

\section{Discussão}

O carrapato bovino, R. (B.) microplus, ocasiona prejuízos pelo estresse imposto aos animais infestados, causando queda no consumo alimentar e, consequente diminuição da produção animal (Oliveira \& Pedrassani, 2017).

Para se estabelecer estratégias de controle adequadas, visando reduzir as perdas de caráter econômico há necessidade do conhecimento do ciclo biológico do $R$. (B.) microplus, sendo também fundamental conhecer a sua epidemiologia (Santos et al., 2013) e a ocorrência de surtos em uma dada região afim de se estabelecer medidas preventivas de controle desse ectoparasita (Amorim et al., 2014).

Métodos de controle como o uso de inimigos naturais, como fungos, bactérias, animais vertebrados e invertebrados estão sendo elaborados e bem vistos quanto ao emprego do controle biológico, tendo como exemplo o método de controle com produto orgânico a base de fungos Metarhizium anisopliae (Camargo et al., 2016).

A utilização de fitoterápicos também tem sido considerada no controle de carrapatos, pois os fitoterápicos promoverem um desenvolvimento mais lento da resistência parasitaria, além de ser um produto mais viável ambientalmente pelo fato de ser biodegradável, diminuir os resíduos oriundos do medicamento no meio ambiente, além de apresentar uma relação de custo benefício melhor que a relação apresentada por produtos agroquímicos (Borges et al., 2011; Pivoto et al., 2010).

Para o controle fitoterápico do $R$. (B.) microplus, destacam-se o uso de extrato hidroalcoólico de eucalipto (Eucalyptus dunni) (Oliveira \& Pedrassani, 2017), extrato hidroalcoólico de citronela (Cymbopogon citratus) (Santos et al., 2013; Agnolin et al., 2014), extrato de neem (Azaridachta indica) (Borges et al., 2011), todos testados in vitro sobre teleógenas, mas com usos e concentrações diferenciadas das realizadas neste estudo. Não foram encontrados relatos do uso de noni em carrapatos.

Vale a pena ressaltar que o tratamento químico comercial de cipermetrina possui em sua composição a associação com citronelal, principal constituinte do extrato hidroalcoólico da citronela que, por sua vez obteve eficácia medicamentosa de $47 \%$, cujos valores encontrados foram semelhantes a Agnolin (2014), onde pode-se observar $48 \%$ de eficácia quando utilizado óleo mineral de citronela a concentração de $2 \%$.

A presença de óleo nos fármacos cipermetrina, neen e citronela, além auxiliar na fixação do princípio ativo no animal, poderiam ter auxiliado no controle do carrapato. Este controle poderia ser explicado pelo fato do óleo provocar asfixia nos carrapatos pelo bloqueio de seus espiráculos, que são aberturas superficiais responsáveis pela respiração dos mesmos (Agnolin et al., 2014). Entretanto, no presente estudo, o óleo de Neen apresentou eficácia de 30\%, com baixa eficácia frente a forma larval do carrapato. 
Os valores encontrados utilizando-se o óleo emulsionável comercial de neen estão em concordância aos obtidos por Broglio-Micheletti et al. (2010), quando utilizaram duas variedades de óleo emulsionável de neen comercial e extrato de semente solubilizados em hexano e etanol, conferindo mortalidade das teleógenas variando de $20 \%$ a $96 \%$, podendo-se observar efeito ascendente da eficiência em função da concentração dos medicamentos.

Tanto o óleo de neen comercial quanto o extrato hidroalcoólico de noni não interferiram na eclosão dos ovos, fator determinante no cálculo de eficiência reprodutiva dos tratamentos. Nas formas larvais, noni e neem também não tiverem efeito esperado nas concentrações utilizadas; entretanto, estudos devem ser conduzidos com concentração progressiva, principalmente para noni. Estudos bem delineados que comprovam a eficácia terapêutica dos compostos bioativos de noni, e que avaliam a sua toxidade são escassos e ainda controversos (Oliveira et al., 2018).

Alternância de princípios, com uso de fitoterápicos de forma preventiva e aplicação da base química para tratamento ou de forma estratégica pode ser útil no controle de carrapato. É interessante a elaboração estratégia de manejo profilático para o controle do carrapato bovino utilizando-se da base química cipermetrina em associação com o extrato hidroalcoólico de citronela, pode aliar a eficiência de ambos os fármacos e otimizando o controle do $R$. (B.) microplus nos seus diferentes estágios evolutivos em sistemas convencionais de produção.

Tratamentos fitoterápicos eficientes dentro dos sistemas de produção é uma prática vantajosa à produção orgânica de proteína animal por se tratarem de fármacos biodegradáveis, atóxicos ao homem, adaptados aos trópicos e não deixarem resíduos nos produtos de origem animal (Borges et al., 2011).

Todos esses fatores aliados a facilidade de manipulação e maior valor agregado devido à grande aceitação por parte do mercado consumidor, tornam a utilização de fármacos fitoterápicos uma alternativa economicamente viável aos sistemas de produção, impulsionando tanto a produção orgânica quanto a agricultura familiar, trazendo maior rentabilidade e qualidade de vida aos pequenos produtores (Fernandes et al., 2012).

O fármaco químico cipermetrina foi capaz de diminuir drasticamente a infestação do carrapato bovino, em áreas não resistentes ao medicamento. Dentre os fitoterápicos, o extrato hidroalcoólico de citronela mostrou-se com eficiência intermediária, superior aos demais extratos de noni e neen em suas respectivas concentrações e que possuem potencial no controle de carrapatos $R$. (B.) microplus.

\section{Referências bibliográficas}

Agnolin, C.A., Olivo, C.J. \& Parra, C. L. C.. (2014). Efeito do óleo de capim limão (Cymbopogon flexuosus Stapf) no controle do carrapato dos bovinos. Revista Brasileira de Plantas Medicinais, 16(1), 77-82.https://doi.org/10.1590/S1516-05722014000100011

Amorim, L. S., Wenceslau, A. A., Carvalho, F. S., Carneiro, P. L. S., \& Albuquerque, G. R. (2014). Bovine babesiosis and anaplasmosis complex: diagnosis and evaluation of the risk factors from Bahia, Brazil. Revista Brasileira de Parasitologia Veterinária, 23(3), 328-336. https://doi.org/10.1590/S198429612014064

Andreotti, R., Garcia, M. V., Matias, J., Barros, J. C., Magalhães, G. M., Ardson, F. A., \& Aguirre, R. A. (2015). Diflubenzuron Effectiveness in Cattle Tick (Rhipicephalus Boophilus microplus) Control in Field Conditions. Pharmaceutica Analytica Acta, 6(373). https://doi.org/10.4172/21532435.1000373

Borges, L. M. F., Sousa, L. A., \& Barbosa, C. S. D. (2011). Perspectives for the use of plant extracts to control the cattle tick Rhipicephalus (Boophilus) microplus. Revista Brasileira de Parasitologia Veterinária, 20(2), 89-96. https://doi.org/10.1590/S1984-29612011000200001

Broglio-Micheletti, S. M. F., Dias, N. S., Valente, E. C. N., Souza, L. A., Lopes, D. O. P., \& Santos, J. M. (2010). Ação de extrato e óleo de nim no controle de Rhipicephalus (Boophilus) microplus (Canestrini, 1887) (Acari: Ixodidae) em laboratório. Revista Brasileira de Parasitologia Veterinária, 19(01), 46-50. https://doi.org/10.4322/rbpv.01901008

Camargo, M. G., Nogueira, M. R. S., Marciano, A. F., Perinotto, W. M. S., Coutinho-Rodrigues, C. J. B., Scott, F. B., Angelo, I. C., Prata, M. C. A., \& Bittencourt, V. R. E. P. (2016). Metarhizium anisopliae for controlling Rhipicephalus microplus ticks under field conditions. Veterinary 
Parasitology, 223, 38-42. https://doi.org/10.1016/j.vetpar.2016.04.014

Correia, A. A. S., Gonzaga, M. L. C., Aquino, A. D., Souza, P. H. M., Figueiredo, R. W., \& Maia, G. A. (2011). Chemical and physical-chemical pulp noni (Morinda citrifolia) grown in the state of Ceará. Alimentos e Nutrição, Araraquara, 22, 606-615.

Drummond, R. O., Ernst, S. E., Trevino, J. L., Gladney, W. J., \& Graham, O. H. (1973). Boophilus annulatus and B. microplus: Laboratory Tests of Insecticides13. Journal of Economic Entomology, 66(1), 130-133. https://doi.org/10.1093/jee/66.1.130

Fernandes, J. I., Verocai, G. G., Ribeiro, F. A., Melo, R. M. P. S., Correia, T. R., Veiga, C. C. P., Vieira, V. P. C., \& Scott, F. B. (2012). Eficácia acaricida de uma emulsão contendo $10 \%$ de óleo de nim (Azadirachta indica) no controle de Psoroptes ovis em coelhos naturalmente infestados. Pesquisa Veterinária Brasileira, 32(12), 1253-1256. https://doi.org/10.1590/S0100-736X2012001200007

Gomes, A., Koller, W. W., \& Barros, A. T. M. (2011). Suscetibilidade de Rhipicephalus (Boophilus) microplus a carrapaticidas em Mato Grosso do Sul, Brasil. Ciência Rural, 41(8), 1447-1452. https://doi.org/10.1590/S0103-84782011005000105

Oliveira, F. C. E., Silva, L. B. P., Abranches, M. V., \& Ferreira, A. A. (2018). Efeitos terapêuticos e adversos do Noni (Morinda Citrifolia L.) na naúde. Revista Saúde \& Ciência Online, 7(3), 107-122. https://doi.org/10.35572/rsc.v7i3.145

Oliveira, M. K. F., \& Pedrassani, D. (2017). Extrato hidroalcoólico de eucalipto, Eucalyptus dunnii, no controle do carrapato bovino, Rhipicephalus (Boophilus) microplus. Revista Acadêmica: Ciência Animal, 15, 41. https://doi.org/10.7213/academica.15.2017.06

Pivoto, F. L., Buzatti, A., Krawczak, F. S., Camillo, G., Sangioni, L. A., Zanetti, G. D., Manfron, M. P., \& Vogel, F. S. F. (2010). Ação acaricida in vitro de Tropaeolum majus sob teleóginas de Rhipicephalus (Boophilus) microplus. Ciência Rural, 40(10), 2141-2145. https://doi.org/10.1590/S0103$\underline{84782010005000170}$

Ríos-Tobón, S., Gutiérrez-Builes, L. A., \& Ríos-Osorio, L. A. (2014). Assessing bovine babesiosis in Rhipicephalus (Boophilus) microplus ticks and 3 to 9-month-old cattle in the middle Magdalena region, Colombia. Pesquisa Veterinária Brasileira, 34(4), 313-319. https://doi.org/10.1590/S0100736X2014000400002

Santos, A. C. G., Rodrigues, O. G., Araújo, L. V. C., Santos, S. B., Guerra, R. M. S. N. C., Feitosa, M. L. T., Teixeira, W. C., \& Santos-Ribeiro, Á. (2006). Uso de extrato de nim no controle de acaríase por Myobia musculi Schranck (Acari: Miobidae) e Myocoptes musculinus Koch (Acari: Listrophoridae) em Camundongos (Mus musculus var. albina L.). Neotropical Entomology, 35(2), 269-272.

Santos, F. C. C., Monteiro, S. G., \& Roll, V. F. (2013). In vitro effect of the association of citronella, Santa Maria herb (Chenopodium ambrosioides) and quassia tincture on cattle tick Rhipicephalus (Boophilus) microplus. Ciência Animal Brasileira, 14(1). https://doi.org/10.5216/cab.v14i1.19393

Sousa, L. A. D., Soares, S. F., Pires Júnior, H. B., Ferri, P. H., \& Borges, L. M. F. (2008). Avaliação da eficácia de extratos oleosos de frutos verdes e maduros de cinamomo (Melia azedarach) sobre Rhipicephalus (Boophilus) microplus (Acari: Ixodidae). Revista Brasileira de Parasitologia Veterinária, 17(1), 36-40. https://doi.org/10.1590/\$1984-29612008000100008

Recebido: 20 de abril, 2020.

Aprovado: 26 de maio, 2020

Disponível online: 18 de setembro, 2020

Licenciamento: Este artigo é publicado na modalidade Acesso Aberto sob a licença Creative Commons Atribuição 4.0 (CC-BY 4.0), a qual permite uso irrestrito, distribuição, reprodução em qualquer meio, desde que o autor e a fonte sejam devidamente creditados. 